\title{
Metagenomic next-generation sequencing for the clinical diagnosis and prognosis of acute respiratory distress syndrome caused by severe pneumonia: a retrospective study
}

\footnotetext{
Peng Zhang ${ }^{1,2}$, Yan Chen ${ }^{3,4}$, Shuyun $\mathrm{Li}^{5}$, Chaoliang Li ${ }^{2}$, Shuang Zhang ${ }^{2}$, Weihao Zheng ${ }^{2}$, Yantang Chen $^{2}$, Jie Ma ${ }^{2}$ , Xin Zhang ${ }^{6}$, Yanming Huang ${ }^{7}$, Shengming Liu ${ }^{\text {Corresp. } 1}$

${ }^{1}$ Department of Respiratory Medicine, The First Affiliated Hospital of Jinan University, Guangzhou, Guangdong, China

2

2 Department of Critical Care Medicine, Jiangmen Central Hospital, Affiliated Jiangmen Hospital of Sun Yat-sen University, Jiangmen, Guangdong, China

3 BGI PathoGenesis Pharmaceutical Technology Co., Ltd, BGI-Shenzhen, Shenzhen, Guangdong, China

4 BGI Wuhan Biotechnology, BGI-Shenzhen, Wuhan, Hubei, China

5 Department of Neurology, Jiangmen Central Hospital, Affiliated Jiangmen Hospital of Sun Yat-sen University, Jiangmen, Guangdong, China

6 Clinical Experimental Center, Jiangmen Central Hospital, Affiliated Jiangmen Hospital of Sun Yat-sen University, Jiangmen, Guangdong, China

7 Department of Respiration Medicine, Jiangmen Central Hospital, Affiliated Jiangmen Hospital of Sun Yat-sen University, Jiangmen, Guangdong, China

Corresponding Author: Shengming Liu

Email address: smliu01@163.com
}

Background. Metagenome Next-Generation Sequencing (mNGS) is a valuable diagnostic tool that can be used for the identification of early pathogens of acute respiratory distress syndrome (ARDS) in severe pneumonia. Little is known about the use of this technology in clinical application and the evaluation of the prognostic value of ARDS. Methods. We performed a retrospective cohort study of patients with ARDS caused by severe pneumonia. Samples were collected from patients in the intensive care unit (ICU) of Jiangmen Central Hospital from January 2018 to August 2019. The no-NGS group was composed of patients given conventional microbiological tests to examine sputum, blood, or bronchoalveolar lavage fluid (BALF). The NGS group was composed of patients tested using mNGS and conventional microbiological tests. We evaluated the etiological diagnostic effect and clinical prognostic value of mNGS in patients with ARDS caused by severe pneumonia. Results. The overall positive rate $(91.1 \%)$ detected by the mNGS method was significantly higher than that of the culture method $(62.2 \%, P=0.001)$, and antibody plus polymerase chain reaction (PCR) $(28.9 \%, \mathrm{P}<0.001)$. Following adjustment of the treatment plan based on microbial testing results, the Acute Physiology and Chronic Health Evaluation-II (APACHE II) score of the NGS group was lower than that of the no-NGS group $7 \mathrm{~d}$ after treatment $(\mathrm{P}<0.05)$. The 28 -day mortality rate of the NGS group was significantly lower than that of the no-NGS group $(P<0.05)$. Longer ICU stay, higher APACHE II score and Sequential Organ Failure Assessment (SOFA) score were risk factors for the death of ARDS, and adjusting the medication regimen based on mNGS results was 
a protective factor. The detection of mNGS can significantly shorten the ICU stay of immunosuppressed patients $(P<0.01)$, shorten the ventilation time $(P<0.01)$, and reduce the ICU hospitalization cost $(P<0.05)$. Conclusions. mNGS is a valuable tool to determine the etiological value of ARDS caused by severe pneumonia to improve diagnostic accuracy and prognosis for this disease. For immunosuppressed patients, mNGS technology can be used in the early stage to provide more diagnostic evidence and guide medications. 
1 Metagenomic next-generation sequencing for the

2 clinical diagnosis and prognosis of acute respiratory

3 distress syndrome caused by severe pneumonia: a

4 retrospective study

5

6

7

8

22

Peng Zhang ${ }^{1,2}$, Yan Chen ${ }^{3,4}$, Shuyun $\mathrm{Li}^{5}$, Chaoliang $\mathrm{Li}^{2}$, Shuang Zhang ${ }^{2}$, Weihao Zheng ${ }^{2}$, Yantang $\mathrm{Chen}^{2}$, Jie $\mathrm{Ma}^{2}$, Xin Zhang ${ }^{6}$, Yanming Huang ${ }^{7}$, Shengming Liu ${ }^{1 *}$

${ }^{1}$ Department of Respiratory Medicine, The First Affiliated Hospital of Jinan University, Guangzhou, Guangdong, China.

${ }^{2}$ Department of Critical Care Medicine, Jiangmen Central Hospital, Affiliated Jiangmen Hospital of Sun Yat-sen University, Jiangmen, Guangdong, China.

${ }^{3}$ BGI PathoGenesis Pharmaceutical Technology Co., Ltd, BGI-Shenzhen, Shenzhen, China.

${ }^{4}$ BGI Wuhan Biotechnology, BGI-Shenzhen, Wuhan, China.

${ }^{5}$ Department of Neurology, Jiangmen Central Hospital, Affiliated Jiangmen Hospital of Sun Yat-sen University, Jiangmen, Guangdong, China.

${ }^{6}$ Clinical Experimental Center, Jiangmen Central Hospital, Affiliated Jiangmen Hospital of Sun Yat-sen University, Jiangmen, Guangdong, China.

${ }^{7}$ Department of Respiration Medicine, Jiangmen Central Hospital, Affiliated Jiangmen Hospital of Sun Yat-sen University, Jiangmen, Guangdong, China.

Corresponding Author:

Shengming Liu $^{1 *}$

Department of Respiratory Medicine, The First Affiliated Hospital of Jinan University, Guangzhou, Guangdong 510630, China.

E-mail: smliu01@,163.com 


\section{Abstract}

Background. Metagenome Next-Generation Sequencing (mNGS) is a valuable diagnostic tool that can be used for the identification of early pathogens of acute respiratory distress syndrome (ARDS) in severe pneumonia. Little is known about the use of this technology in clinical application and the evaluation of the prognostic value of ARDS.

Methods. We performed a retrospective cohort study of patients with ARDS caused by severe pneumonia. Samples were collected from patients in the intensive care unit (ICU) of Jiangmen Central Hospital from January 2018 to August 2019. The no-NGS group was composed of patients given conventional microbiological tests to examine sputum, blood, or bronchoalveolar lavage fluid (BALF). The NGS group was composed of patients tested using mNGS and conventional microbiological tests. We evaluated the etiological diagnostic effect and clinical prognostic value of mNGS in patients with ARDS caused by severe pneumonia.

Results. The overall positive rate $(91.1 \%)$ detected by the mNGS method was significantly higher than that of the culture method $(62.2 \%, P=0.001)$, and antibody plus polymerase chain reaction $(\mathrm{PCR})(28.9 \%, P<0.001)$. Following adjustment of the treatment plan based on microbial testing results, the Acute Physiology and Chronic Health Evaluation-II (APACHE II) score of the NGS group was lower than that of the no-NGS group $7 \mathrm{~d}$ after treatment $(P<0.05)$. The 28-day mortality rate of the NGS group was significantly lower than that of the no-NGS group $(P<0.05)$. Longer ICU stay, higher APACHE II score and Sequential Organ Failure Assessment (SOFA) score were risk factors for the death of ARDS, and adjusting the medication regimen based on mNGS results was a protective factor. The detection of mNGS can significantly shorten the ICU stay of immunosuppressed patients $(P<0.01)$, shorten the ventilation time $(P<0.01)$, and reduce the ICU hospitalization cost $(P<0.05)$.

Conclusions. mNGS is a valuable tool to determine the etiological value of ARDS caused by severe pneumonia to improve diagnostic accuracy and prognosis for this disease. For immunosuppressed patients, mNGS technology can be used in the early stage to provide more diagnostic evidence and guide medications.

\section{Introduction}

Acute respiratory distress syndrome (ARDS) is typically caused by infections, such as pneumonia (Saguil \& Fargo 2012). Failure of timely and effective treatment will lead to multiple organ failure and death. Approximately, 31\% (Griffiths et al. 2019)of patients with ARDS are admitted to the intensive care unit (ICU), with a mortality rate of $19.7 \%-57.7 \%$ (Bein et al. 2016; Bellani et al. 2016; Griffiths et al. 2019). ARDS survivors are at greater risk of cognitive decline, depression, post-traumatic stress disorder, and persistent skeletal muscle weakness (Herridge et al. 2016; Herridge et al. 2011), bringing a great economic burden to families and society. Early pathogen identification and clinical intervention are critical for ARDS patients to reduce mortality and improve prognosis (Lee 2017). 
Conventional microbiological testing includes bacterial/fungal culture, polymerase chain reaction (PCR) nucleic acid hybridization, and serological antibody testing. The turn-around time (TAT) of bacterial/fungal cultures is long (3-5 d), and the positive rate is low (Miao et al. 2018). PCR nucleic acid hybridization requires pre-screening of microbial pathogens and designing specific primers/probes, but detection types are limited (Spackman et al. 2002). There is a window period that cannot be accurately identified by the serological antibody detection (Rajapaksha et al. 2019). Metagenome Next-Generation Sequencing (mNGS) was first used to diagnose a central nervous system (CNS) infection of Leptospira in 2014 (Wilson et al. 2014). This emerging diagnostic technology can quickly detect all nucleic acids in specimens of different sample types in one test, including blood, respiratory tract, CNS, and focal tissue (Guan et al. 2016; Guo et al. 2019; Long et al. 2016; Miao et al. 2018). Metagenome NGS technology has been successfully used clinically for rapid identification of pathogens in ARDS patients with pneumonia (Fischer et al. 2014) and can be used in clinical diagnosis and drug decision-making of severe pneumonia (Yang et al. 2019).

Different physiological indicators are crucial to the development and prognosis of ARDS in patients. Reduction of platelet count following ICU admission, age, body mass index, immunocompromised status, prone positioning, days of mechanical ventilation, disease score, elevated cardiac troponin $\mathrm{T}$, extent of endothelial injury, low $\mathrm{PaO}_{2} / \mathrm{FiO}_{2}$ ratio, and different clinical intervention treatment options (Chen \& Ware 2015) affects the prognosis of patients with ARDS. Prior analysis of the prognosis of patients with ARDS using multiple Cox regression models found that late-onset moderate to severe ARDS was associated with adverse outcomes (Zhang et al. 2017). However, the effect of mNGS technology on the prognosis of ARDS patients is unknown.

Currently, the clinical application of mNGS in ARDS appears predominantly in case reports or small-scale cohort studies. There is an urgent need to review the practical application of mNGS technology in ARDS patients, and assess its prognostic value. Thus, this study summarizes clinical information via retrospective analysis, and evaluates the clinical prognosis of ARDS by mNGS technology application.

\section{Materials \& Methods}

\section{Ethical approval and consent}

The protocol used in this retrospective study was reviewed and approved by the Ethical Review Committee of Jiangmen Central Hospital (No: 2019-15). Patient's informed consent was obtained from patients or their next of kin.

\section{Study participants}

A retrospective analysis was conducted on all ARDS cases resulting from severe pneumonia in patients 18 years and older, admitted to the ICU at Jiangmen Central Hospital from January 2018 to August 2019. For our study, ARDS was diagnosed according to the 2012 Berlin definition of the disease (Ards Definition Task Force et al. 2012). Patients were excluded from 
115 the study if their ARDS was not caused by severe pneumonia or if they did not follow through 116 with their treatment for any reason.

117 All patients were endotracheally intubated, mechanically ventilated, and underwent a 118 fiberoptic bronchoscopy to obtain clinical specimens for microbial testing. Patients were

119

120

121

122

123

124

125

126

127

128

129

130

131

132

133

134

135

136

137

138

139

140

141

142

143

144

145

146

147

148

149

150

151

152

153

154 included in the NGS group when informed consent was provided for testing; those who were not tested by mNGS were grouped into the no-NGS group. Owing to the cost of mNGS, only DNA sequencing was performed. Samples of bronchoalveolar lavage fluid (BALF) were acquired from patients in the NGS group and sent for pathogen testing at BGI Clinical Laboratories (Shenzhen) Co., Ltd. Once the laboratory received the samples, nucleic acid extraction, library construction, high-throughput sequencing, bioinformatics analysis, and pathogen data interpretation were performed according to previous studies (Miao et al. 2018).

\section{Microbiological testing}

Both groups were tested using the same conventional method (routine culture + serum antibody + PCR). The NGS group used $\mathrm{mNGS}+$ routine culture + serum antibody $+\mathrm{PCR}$, while the no-NGS group used routine culture + serum antibody + PCR. Pathogenic microbes that cause severe pneumonia are typically bacteria, fungi, or viruses. Restricted by inspection conditions of the hospital, serum antibody and PCR nucleic acid detection could only detect some special pathogens and viruses that were clinically difficult to culture, as a supplement to routine culture. The serum antibody included Mycoplasma pneumoniae, Chlamydia pneumoniae, Coxsackie virus, cytomegalovirus, influenza A, influenza B, respiratory syncytial virus, and parainfluenza virus. PCR nucleic acids included Legionella pneumophila, Mycoplasma pneumoniae, Chlamydia pneumoniae, Mycobacterium tuberculosis, influenza A, and influenza B.

\section{Clinical treatment}

All patients underwent empirical antimicrobial treatment according to the Chinese Adult Community-Acquired Pneumonia Diagnosis and Treatment Guide (Cao et al. 2018) Error! Reference source not found.and the Chinese Adult Hospital-Acquired Pneumonia and Ventilator-associated Pneumonia Diagnosis Guide (Department of Infectious Diseases Chinese Medical Association Respiratory Branch 2018), combined with respiratory infection indicators and imaging. All patients were treated with mechanical ventilation according to the ARDS ventilation guidelines (Bein et al. 2016; Griffiths et al. 2019). The no-NGS patients were treated with an antimicrobial regimen based on the results of conventional microbiological tests. The antimicrobial regimen of NGS patients were adjusted case-by-case according to mNGS results.

\section{Information collection and analysis}

Patient data included age, gender, basic disease, laboratory test results before treatment, ventilator parameters, conventional microbiological tests, serum biomarkers, ICU special treatment data, APACHE II, and SOFA scores. Data were collected and compared between the two groups. The primary outcome was measured by a 28 -day all-cause mortality. Secondary outcomes were measured as the length of stay in the ICU, duration of mechanical ventilation, duration of extracorporeal membrane oxygenation (ECMO), duration of prone ventilation positioning, and ICU treatment costs. Patients that showed signs of immunosuppression were 
155

156

157

158

159

160

161

162

163

164

165

166

167

168

169

170

171

172

173

174

175

176

177

178

179

180

181

182

183

184

185

186

187

188

189

190

191

192

193

194

selected from both groups and their prognosis compared using the same aforementioned outcomes. Cox regression analysis was conducted to analyze risk factors for ARDS prognosis.

The mNGS results were compared with those of conventional microbiological tests in the NGS group.

\section{Statistical Analysis}

The t-test was used to determine normal distribution and uniformity of variance. The Wilcoxon rank test was used to calculate variance of measured data that were not normally distributed or had variance homogeneity. The chi-square test was used to calculate the difference between both groups. All statistical analyses were conducted using GraphPad 5.0 and R3.4.4 software. $P<0.05$ was considered statistically significant.

\section{Results}

\section{Sample and patient characteristics}

A total of 105 patients with ARDS caused by severe pneumonia were screened in this study and 10 patients were excluded based on exclusion criteria. Fourty two patients were placed into the NGS group and 53 patients in the no-NGS group. Three patients in the NGS group had two mNGS tests performed and a total of 45 BALF samples were sent for mNGS.

Patient demographics, characteristic baselines, and ICU special treatments in the NGS and noNGS groups were shown in Tables 1, 2, and 3, respectively. There were no significant differences in age, gender, basic disease, laboratory test results before treatment, ventilator parameters, APACHE II and SOFA scores before treatment, and incidences of special treatment in the ICU between both groups $(P>0.05)$.

\section{Comparison of outcomes between NGS and no-NGS groups}

There was a significant difference in the 28-day all-cause mortality between both groups $(P=$ 0.006) (Table 4). The 28-day survival was significantly higher in the NGS group than in the noNGS group (Hazard Ratio $=2.41,95 \%$ CI: $1.21-4.17, P=0.01$ ) (Figure 1). There was no significant difference in the length of stay in the ICU, duration of mechanical ventilation, ECMO, prone position ventilation, or the cost of the ICU stay between both groups $(P>0.05)$ (Table 4).

\section{Prognosis of ARDS patients}

Cox univariate analysis was performed on all factors and Cox multivariate analysis was performed with variates which were $P<0.2$ of the Cox univariate analysis (Supplemental table). The NGS or no-NGS group, length of stay in ICU, and APACHE II and SOFA scores before treatment were risk factors in patients with ARDS caused by severe pneumonia. The NGS group patients had a better prognosis than that of the no-NGS group patients $(P=0.005)$. A shorter stay in the ICU $(P=0.037)$, and lower APACHE II $(P=0.016)$ and SOFA scores before treatment $(P$ $=0.003$ ) had a better prognosis (Table 5).

\section{Comparison of mNGS results and culture results in the NGS group}

The current research showed that the mNGS test can detect more pathogens than the culture method. We analyzed the consistency of pathogens identified by both techniques. The test results 
195

196

197

198

199

200

201

202

203

204

205

206

207

208

209

210

211

212

213

214

215

216

217

218

219

220

221

222

223

224

225

226

227

228

229

230

231

232

233

were considered to be consistent when the pathogens identified by mNGS were the same as the pathogens obtained from culture. The test results were also considered consistent if mNGS identified more pathogens than the culture method. The result was partially consistent when pathogens identified by both methods were partially congruent. The results were considered inconsistent when pathogens identified by both methods varied completely. Identified pathogens $(31.1 \%)$ in the NGS group were consistent, $15.6 \%$ were partially consistent, and $53.3 \%$ were completely inconsistent. In the inconsistent ones, $62.5 \%$ were negative for the culture method, while $8.3 \%$ were negative for mNGS, and 29.2\% were mismatched (Figure 2).

\section{Comparison metagenomic of NGS results and conventional microbiological tests}

Some special pathogens were difficult to obtain via culture. Therefore, Legionella, Tuberculosis, Mycoplasma/Chlamydia, parasites, K. spores, etc. were defined as such. Severe pneumonia is not caused by a single pathogen and is typically accompanied by co-infections. A co-infection is defined as a non-single pathogenic infection, such as bacteria + fungi/bacteria + virus/fungi + virus/bacteria + fungi + virus.

The positive rate of mNGS virus detection was lower than that of serum antibody detection plus PCR (6.7\% vs. $26.7 \%, P=0.021)$. In this study, mNGS only performed DNA sequencing and could only detect DNA viruses, whereas viruses identified by serological antibody detection and PCR were RNA viruses, such as influenza A and influenza B. mNGS was significantly better at detecting bacteria than serological antibody testing plus PCR $(24.4 \% v s .0 \%, P=0.001)$. Further, mNGS was able to detect specific pathogens better than the culture method $(22.2 \% v s$. $0 \%, P=0.001)$ and serological antibody testing plus PCR $(22.2 \% v s .2 .2 \%, P=0.007)$. Additionally, mNGS was significantly better at the identification of co-infections than serological antibody tests plus PCR $(26.7 \%$ vs. $0 \%, P<0.001)$. Finally, mNGS proved to be significantly better at identifying pathogens than the culture method $(91.1 \%$ VS $62.2 \%, P=$ $0.001)$ and serological antibody testing plus PCR $(91.1 \%$ vs. $28.9 \%, P<0.001)$ (Table 6$)$.

\section{Clinical medication guidance between NGS and no-NGS groups}

In the NGS group, 30 patients (71.4\%) did not cover all the microbial detected by mNG in the initial empirical antimicrobial treatment. Thus, antimicrobial regimen needs to be modified accordingly based on the mNGS results. In the no-NGS group, empirical antimicrobial treatment that could not cover the detected microbials was found in 23 patients $(43.4 \%)$, according to the results of traditional microbiological testing, and they were necessary to adjust the anti-infection program (Figure 3). Following adjustment of the anti-infective regimen, we continuously observed APACHE II and SOFA scores for both groups of patients for 7 days and found that the NGS group had a lower APACHE II score than the no-NGS group, 7 days after treatment $(P=$ 0.041) (Figure 4).

\section{Immunosuppressed patients}

Clinical features of immunosuppressed patients were complicated. A total of 21 immunosuppressed patients were enrolled in our study, eight were subjected to mNGS pathogen detection, and 13 did not undergo mNGS. Three cultures were positive in the NGS group, 
234 consistent with pathogens identified by mNGS, including five $P$. jirovecii, one Rhizopus, one

235

236

237

238

239

240

241

242

243

244

245

246

247

248

249

250

251

252

253

254

255

256

257

258

259

260

261

262

263

264

265

266

267

268

269

270

271

272

Cryptococcus, and one human herpesvirus; six were co-infections.

In the no-NGS group, nine cases were positive for culture, and two $S$. maltophilia, two $A$. baumannii, one $S$. aureus, four Candida, and one Aspergillus were detected. Four cases had multi-drug resistant bacteria. There was no significant difference in the 28-day all-cause mortality between the two groups $(37.5 \%$ vs.53.8\%, $P=0.659)$. However, there were significant differences in the length of stay in the ICU $(P=0.023)$, duration of mechanical ventilation $(P=$ $0.030)$, and cost of the stay in the ICU $(P=0.004)$ between both groups of immunosuppressed patients (Figure 5).

\section{Discussion}

ARDS caused by severe pneumonia is critical and progresses rapidly. Common microbial infection includes those of bacteria, fungi, and viruses while some are co-infections (Lee 2017). Patients usually require broad-spectrum anti-infection treatment, and then, further adjust to targeted anti-infection treatment based on microbial detection results of. Therefore, it is critical to determine the type of microbial infection for ARDS treatment caused by severe pneumonia.

This study compared the effectiveness of mNGS with traditional microbiological testing methods of the NGS group. Firstly, mNGS was faster, taking an average of 2 days from sending samples to receiving reports, whereas routine culture requires at least 3-5 days. Secondly, the overall positive rate $(91.1 \%)$ of $\mathrm{mNGS}$ was significantly higher than that of culture $(62.2 \%, P=$ $0.001)$ and antibody plus PCR $(28.9 \%, P<0.001)$. As all patients included were diagnosed with severe pneumonia, the positive rate of $\mathrm{mNGS}$ and culture of lower respiratory tract specimens were higher than that of usual detection. Thirdly, the positive rate of mNGS detection of specific pathogens $(22.2 \%)$ was higher than that of culture $(0 \%, P=0.001))$ and antibody plus PCR $(2.2 \%, P=0.007)$. This conclusion was consistent with a previous study by Qi et al. (Qi et al. 2019) in that the positive of mNGS was much higher than that of culture, and rare pathogens could be detected. In addition, we analyzed the consistency between mNGS and culture, 31.1\% of identified pathogens in the NGS group were consistent, $15.6 \%$ were partially consistent, and $53.3 \%$ were completely inconsistent. In the inconsistent ones, $62.5 \%$ were negative for culture, while only $8.3 \%$ were negative for mNGS. The advantages of mNGS detection compared with traditional detection were confirmed.

By comparing the prognosis of patients between the NGS group and the no-NGS group, it was found that the 28-day mortality rate of the NGS group was significantly lower than that of the no-NGS group $(P<0.05)$ (Table 4$)$. There was no difference in ICU hospitalization time, mechanical ventilation time, ECMO time, prone position ventilation time, and ICU treatment costs between the two groups (Table 4). This conclusion was consistent with the study of Xie et al. (Xie et al. 2019). They analyzed 178 patients with severe pneumonia and combined mNGS results to guide treatment. The 28-day and 90-day survival rates of severe pneumonia patients were improved. The 90 -day survival rate increased from $57.7 \%$ to $83.3 \%$.

Peer] reviewing PDF | (2020:02:45653:2:0:NEW 24 Jun 2020) 
273

274

275

276

277

278

279

280

281

282

283

284

285

286

287

288

289

290

291

292

293

294

295

296

297

298

299

300

301

302

303

304

305

306

307

308

309

310

311

312

In this study, clinicians assisted clinical diagnosis through comprehensive microbial testing; the empirical medication of $71.4 \%$ of patients in the NGS group did not cover clinically diagnosed microbial infections, whose anti-infection treatment should be adjusted based on mNGS results. In the no-NGS group, $43.4 \%$ of patients required adjustment of the empirical anti-infection regimen. Due to faster and more effective adjustment of the anti-infection regimen, it was found that APACHE II scores in the NGS group were lower than those in the no-NGS group $7 \mathrm{~d}$ after treatment $(P=0.041$, Figure 4$)$. This means that the mNGS test results have a positive effect on clinical medication guidance. Moreover, a multiple Cox regression analysis was conducted for assessment of prognostic factors and found that a longer stay in ICU, high APACHE II score, and high SOFA score were risk factors for ARDS death, and the application of mNGS for clinical pathogen detection was a protective factor. It was shown that the higher the APACHE II and SOFA scores of sepsis patients, the worse the prognosis (Innocenti et al. 2014; Jones et al. 2009), which is consistent with our results.

In addition, studies have shown that immunosuppressed patients were prone to co-infection. Metagenomics NGS technology has distinct advantages in detecting co-infection pathogens (Parize et al. 2017). In this study, mNGS detected specific pathogens that were difficult to culture in immunosuppressed patients, including Pneumocystis, Rhizopus, Cryptococcus, and viruses. Although the mortality rate of the NGS group was lower than that of the no-NGS group, the difference in the prognostic analysis of immunosuppressed patients was not statistically significant $(37.5 \%$ vs $53.8 \%, P=0.659)$, and may be related to the small sample size. Moreover, we found that mNGS technology can significantly shorten the length of stay in the ICU of immunosuppressed patients, shorten the ventilation time, and reduce the cost in ICU $(P<0.05)$. From the economics and clinical prognosis, immunosuppressed patients were more suitable for mNGS technology application in the early clinical stage to assist clinical diagnosis and drug decision-making.

Limitations to the use of mNGS technology exist, despite its widespread use. There is no authoritative guide to the interpretation of the mNGS report. Detection of a broad spectrum of pathogens by mNGS has blunted the diagnosis of pathogenicity resulting in the inability to distinguish between background, colonization and microbial infection, and pollution (Simner et al. 2018). Better technology needs to be developed for mNGS to be used successfully in clinical applications. The use of mNGS in clinical applications will: (1) achieve a faster diagnosis of pathogens and obtain information on drug resistance of related pathogens; (2) identify microbial colonization or infection through monitoring the patient's immune response, which will eventually curb bacterial resistance, achieve a rational application of antibiotics, and ultimately reduce the economic and social burden of infectious diseases; (3) lower the cost of the mNGS test with the development of technology, so that more patients will benefit.

Our research also has certain limitations. Firstly, our mNGS only performed DNA sequencing and did not perform RNA sequencing; therefore, the information of RNA virus and microbial transcriptome alterations were missing, resulting in the positive rate of $\mathrm{mNGS}$ virus detection being lower than serum antibody plus PCR $(6.7 \%$ vs $26.7 \%, P=0.021)$. Secondly, restricted by 
313 the inspection conditions of the hospital, PCR detection only included some RNA viruses, such 314 as influenza A and influenza B. Additionally, the prognostic analysis was affected by several 315 clinical factors and sample size of this study was not large, resulting in some data inconsistency. 316 For example, the mortality rate between the two groups was significantly different, but that of

317 the immunosuppressed patients was not. There was no difference in ICU stay, cost, and

318 ventilation time between the two groups, but there was a difference between the two groups of

319 immunosuppressed patients.

320

321

\section{Conclusions}

322

mNGS technology is valuable for the diagnosis, treatment and prognosis of ARDS caused by

323

324

325

326

327

328

329

330

331

332

333

334

335

336

337

338

339

340

341

342

343

344

345

346

347

348

349

350 severe pneumonia. mNGS technology is superior to conventional microbiological tests for the detection of special pathogens and co-infections. mNGS technology harbors great potential for clinical infection. Further research should include a larger sample size, involving multi-center, prospective, and controlled studies, which will help us better understand the clinical experience summary and prognostic value of mNGS detection in ARDS caused by severe pneumonia.

\section{Additional Information and Declarations}

\section{Competing Interests}

Yan Chen is an employee of BGI Genomics. The authors declare that they have no competing interests.

\section{Author Contributions}

All authors had access to the full dataset (including statistical reports and tables) and take responsibility for the integrity of the data and the accuracy of the data analysis. All authors have read and approved the final manuscript.

Shengming Liu and Yanming Huang conceived and designed the study. They reviewed and approved the final report.

Peng Zhang, Yan Chen, Shuyun Li, Chaoliang Li, Shuang Zhang, Weihao Zheng, Yantang Chen, Jie Ma, and Xin Zhang were involved in the case and sample collection, analysis, interpretation of data and wrote the first draft of the manuscript.

\section{Data Availability}

The datasets generated and analyzed during the current study are available in the (Figshare) repository (https://figshare.com/articles/data_xlsx/10308617). The data showed 95 patients with ARDS caused by severe pneumonia.

\section{Funding}


351

352

353

354

355

356

357

358

359

360

361

362

363

364

365

366

367

368

369

370

371

372

373

374

375

376

377

378

379

380

381

382

383

384

385

386

387

388

389

390

391

392

393

394

This study was supported by the grants from the National Natural Science Foundation of China (81802918), National Science and Technology Major Project of China (2018ZX10305409-001001), the Science and Technology Project of Guangdong Province (2019A1515011565, 2018A030310007), the Science Foundation of Guangdong Province Bureau of Traditional Chinese Medicine (20181273) and the Medical Science Foundation of Jiangmen Central Hospital (J201801). The funder had no role in the study design, collection, analysis, and interpretation of data, and in writing the manuscript.

\section{Acknowledgements}

We would like to thank Jacqueline Thai (Peer J) and Editage (www.editage.cn) for English language editing.

\section{References}

Ards Definition Task Force O, Ranieri VM, Rubenfeld GD, Thompson BT, Ferguson ND, Caldwell E, Fan E, Camporota L, and Slutsky AS. 2012. Acute respiratory distress syndrome: the Berlin Definition. JAMA 307:2526-2533. 10.1001/jama.2012.5669

Bein T, Grasso S, Moerer O, Quintel M, Guerin C, Deja M, Brondani A, and Mehta S. 2016. The standard of care of patients with ARDS: ventilatory settings and rescue therapies for refractory hypoxemia. Intensive Care Med 42:699-711. 10.1007/s00134-016-4325-4

Bellani G, Laffey JG, Pham T, Fan E, Brochard L, Esteban A, Gattinoni L, van Haren F, Larsson A, McAuley DF, Ranieri M, Rubenfeld G, Thompson BT, Wrigge H, Slutsky AS, Pesenti A, Investigators LS, and Group ET. 2016. Epidemiology, Patterns of Care, and Mortality for Patients With Acute Respiratory Distress Syndrome in Intensive Care Units in 50 Countries. JAMA 315:788-800. 10.1001/jama.2016.0291

Cao B, Huang Y, She DY, Cheng QJ, Fan H, Tian XL, Xu JF, Zhang J, Chen Y, Shen N, Wang H, Jiang M, Zhang XY, Shi Y, He B, He LX, Liu YN, and Qu JM. 2018. Diagnosis and treatment of community-acquired pneumonia in adults: 2016 clinical practice guidelines by the Chinese Thoracic Society, Chinese Medical Association. Clin Respir J 12:13201360. $10.1111 /$ crj. 12674

Chen W, and Ware LB. 2015. Prognostic factors in the acute respiratory distress syndrome. Clin Transl Med 4:65. 10.1186/s40169-015-0065-2

Department of Infectious Diseases Chinese Medical Association Respiratory Branch O. 2018. Chinese Adult Hospital Acquired Pneumonia and Ventilator-associated Pneumonia Diagnosis and Treatment Guide (2018 Edition). Chinese Journal of Tuberculosis and Respiratory Diseases 41:255-280.

Fischer N, Rohde H, Indenbirken D, Gunther T, Reumann K, Lutgehetmann M, Meyer T, Kluge S, Aepfelbacher M, Alawi M, and Grundhoff A. 2014. Rapid metagenomic diagnostics for suspected outbreak of severe pneumonia. Emerg Infect Dis 20:1072-1075. 10.3201/eid2006.131526

Griffiths MJD, McAuley DF, Perkins GD, Barrett N, Blackwood B, Boyle A, Chee N, Connolly B, Dark P, Finney S, Salam A, Silversides J, Tarmey N, Wise MP, and Baudouin SV. 2019. Guidelines on the management of acute respiratory distress syndrome. BMJ Open Respir Res 6:e000420. 10.1136/bmjresp-2019-000420

Guan H, Shen A, Lv X, Yang X, Ren H, Zhao Y, Zhang Y, Gong Y, Ni P, Wu H, Zhu Y, and

Peer) reviewing PDF | (2020:02:45653:2:0:NEW 24 Jun 2020) 
395

396

397

398

399

400

401

402

403

404

405

406

407

408

409

410

411

412

413

414

415

416

417

418

419

420

421

422

423

424

425

426

427

428

429

430

431

432

433

434

435

436

437

438

439

440

Cui L. 2016. Detection of virus in CSF from the cases with meningoencephalitis by nextgeneration sequencing. J Neurovirol 22:240-245. 10.1007/s13365-015-0390-7

Guo LY, Feng WY, Guo X, Liu B, Liu G, and Dong J. 2019. The advantages of next-generation sequencing technology in the detection of different sources of abscess. $J$ Infect 78:75-86. 10.1016/j.jinf.2018.08.002

Herridge MS, Moss M, Hough CL, Hopkins RO, Rice TW, Bienvenu OJ, and Azoulay E. 2016. Recovery and outcomes after the acute respiratory distress syndrome (ARDS) in patients and their family caregivers. Intensive Care Med 42:725-738. 10.1007/s00134-016-4321-8

Herridge MS, Tansey CM, Matte A, Tomlinson G, Diaz-Granados N, Cooper A, Guest CB, Mazer CD, Mehta S, Stewart TE, Kudlow P, Cook D, Slutsky AS, Cheung AM, and Canadian Critical Care Trials G. 2011. Functional disability 5 years after acute respiratory distress syndrome. $N$ Engl J Med 364:1293-1304. 10.1056/NEJMoa1011802

Innocenti F, Bianchi S, Guerrini E, Vicidomini S, Conti A, Zanobetti M, and Pini R. 2014. Prognostic scores for early stratification of septic patients admitted to an emergency department-high dependency unit. Eur J Emerg Med 21:254-259.

10.1097/MEJ.0000000000000075

Jones AE, Trzeciak S, and Kline JA. 2009. The Sequential Organ Failure Assessment score for predicting outcome in patients with severe sepsis and evidence of hypoperfusion at the time of emergency department presentation. Crit Care Med 37:1649-1654. 10.1097/CCM.0b013e31819def97

Lee KY. 2017. Pneumonia, Acute Respiratory Distress Syndrome, and Early Immune-Modulator Therapy. Int J Mol Sci 18. 10.3390/ijms18020388

Long Y, Zhang Y, Gong Y, Sun R, Su L, Lin X, Shen A, Zhou J, Caiji Z, Wang X, Li D, Wu H, and Tan H. 2016. Diagnosis of Sepsis with Cell-free DNA by Next-Generation Sequencing Technology in ICU Patients. Arch Med Res 47:365-371. 10.1016/j.arcmed.2016.08.004

Miao Q, Ma Y, Wang Q, Pan J, Zhang Y, Jin W, Yao Y, Su Y, Huang Y, Wang M, Li B, Li H, Zhou C, Li C, Ye M, Xu X, Li Y, and Hu B. 2018. Microbiological Diagnostic Performance of Metagenomic Next-generation Sequencing When Applied to Clinical Practice. Clin Infect Dis 67:S231-S240. 10.1093/cid/ciy693

Parize P, Muth E, Richaud C, Gratigny M, Pilmis B, Lamamy A, Mainardi JL, Cheval J, de Visser L, Jagorel F, Ben Yahia L, Bamba G, Dubois M, Join-Lambert O, Leruez-Ville M, Nassif X, Lefort A, Lanternier F, Suarez F, Lortholary O, Lecuit M, and Eloit M. 2017. Untargeted next-generation sequencing-based first-line diagnosis of infection in immunocompromised adults: a multicentre, blinded, prospective study. Clin Microbiol Infect 23:574 e571-574 e576. 10.1016/j.cmi.2017.02.006

Qi C, Hountras P, Pickens CO, Walter JM, Kruser JM, Singer BD, Seed P, Green SJ, and Wunderink RG. 2019. Detection of respiratory pathogens in clinical samples using metagenomic shotgun sequencing. J Med Microbiol 68:996-1002. 10.1099/jmm.0.000968

Rajapaksha P, Elbourne A, Gangadoo S, Brown R, Cozzolino D, and Chapman J. 2019. A review of methods for the detection of pathogenic microorganisms. Analyst 144:396-411. $10.1039 / \mathrm{c} 8 \mathrm{an} 01488 \mathrm{~d}$

Saguil A, and Fargo M. 2012. Acute respiratory distress syndrome: diagnosis and management. Am Fam Physician 85:352-358.

Simner PJ, Miller S, and Carroll KC. 2018. Understanding the Promises and Hurdles of Metagenomic Next-Generation Sequencing as a Diagnostic Tool for Infectious Diseases.

Peer] reviewing PDF | (2020:02:45653:2:0:NEW 24 Jun 2020) 
441

442

443

444

445

446

447

448

449

450

451

452

453

454

455

456

457

458

459

460

461

462

463

464

465

466

467

468

469

470

471

472

473

474

475

476

477

478

479

480

481

482

Clin Infect Dis 66:778-788. 10.1093/cid/cix881

Spackman E, Senne DA, Myers TJ, Bulaga LL, Garber LP, Perdue ML, Lohman K, Daum LT, and Suarez DL. 2002. Development of a real-time reverse transcriptase PCR assay for type A influenza virus and the avian $\mathrm{H} 5$ and $\mathrm{H} 7$ hemagglutinin subtypes. J Clin Microbiol 40:3256-3260. 10.1128/jcm.40.9.3256-3260.2002

Wilson MR, Naccache SN, Samayoa E, Biagtan M, Bashir H, Yu G, Salamat SM, Somasekar S, Federman S, Miller S, Sokolic R, Garabedian E, Candotti F, Buckley RH, Reed KD, Meyer TL, Seroogy CM, Galloway R, Henderson SL, Gern JE, DeRisi JL, and Chiu CY. 2014. Actionable diagnosis of neuroleptospirosis by next-generation sequencing. $N$ Engl J Med 370:2408-2417. 10.1056/NEJMoa1401268

Xie Y, Du J, Jin W, Teng X, Cheng R, Huang P, Xie H, Zhou Z, Tian R, Wang R, and Feng T. 2019. Next generation sequencing for diagnosis of severe pneumonia: China, 2010-2018. J Infect 78:158-169. 10.1016/j.jinf.2018.09.004

Yang L, Haidar G, Zia H, Nettles R, Qin S, Wang X, Shah F, Rapport SF, Charalampous T, Methe B, Fitch A, Morris A, McVerry BJ, O'Grady J, and Kitsios GD. 2019. Metagenomic identification of severe pneumonia pathogens in mechanically-ventilated patients: a feasibility and clinical validity study. Respir Res 20:265. 10.1186/s12931-0191218-4

Zhang R, Wang Z, Tejera P, Frank AJ, Wei Y, Su L, Zhu Z, Guo Y, Chen F, Bajwa EK, Thompson BT, and Christiani DC. 2017. Late-onset moderate to severe acute respiratory distress syndrome is associated with shorter survival and higher mortality: a two-stage association study. Intensive Care Med 43:399-407. 10.1007/s00134-016-4638-3

\section{FIGURE LEGENDS}

\section{Figure 1. Analysis of 28-day survival curves of patients in the NGS group and no-NGS} group.

The 28-day survival was significantly higher in the NGS group than in the no-NGS group $(\mathrm{HR}=2.41,95 \% \mathrm{CI}: 1.21-4.17, P=0.01)$

Figure 2 . The consistent analysis comparing culture and mNGS pathogen detection in the NGS group.

Identified pathogens (31.1\%) in the NGS group were consistent, $15.6 \%$ were partially consistent, and $53.3 \%$ were completely inconsistent. In the inconsistent ones, $62.5 \%$ were negative for the culture method, while $8.3 \%$ were negative for mNGS, and $29.2 \%$ were mismatched.

\section{Figure 3. Coverage spectrum of empirical antimicrobial therapy for pathogen detection} results in two groups.

A: In the NGS group, 30 patients (71.4\%) did not cover all the microbial detected by mNGS in the initial empirical antimicrobial treatment. Thus, antimicrobial regimen needs to be modified accordingly based on the mNGS results. B: In the no-NGS group, empirical antimicrobial treatment that could not cover the detected microbials was found in 23 patients $(43.4 \%)$, according to the results of traditional microbiological testing, and they were necessary to adjust the anti-infection program.

Figure 4. APACHE II and SOFA scores of the two groups. 
483 A: The NGS group had a lower APACHE II score than that in the no-NGS group after 7 days of 484 treatment $(P=0.041)$. B: There was no significant difference in SOFA score during 7 days 485 between two groups $(P>0.05)$.

486 Figure 5. Clinical data of 21 immunosuppressed patients with NGS and no-NGS were 487 compared.

488 The NGS group had shorter length of stay in the ICU (A) $(P=0.023)$, shorter ventilation time (B) $489(P=0.030)$, and less cost in ICU $(C)(P=0.004)$ than those in the no-NGS group of 490 immunosuppressed patients. 


\section{Table $\mathbf{1}$ (on next page)}

Patient characteristics and baseline of two groups.

There were no any differences in age, sex ratio, basis disease between two groups $(P>$ $0.05)$. 
1 Table 1. Patient characteristics and baseline of the two groups.

\begin{tabular}{lccc}
\hline & NGS (n=42) & no-NGS (n=53) & $P$ value \\
\hline Age (yr) & & & \\
$\geq 60, \mathrm{n}(\%)$ & $21(50.0)$ & $20(62.3)$ & 0.231 \\
$\quad<60, \mathrm{n}(\%)$ & & & \\
Gender & $31(50.0)$ & $38(71.7)$ & 0.819 \\
$\quad$ Male, n (\%) & $11(26.2)$ & $15(28.3)$ & \\
$\quad$ Female, n (\%) & & $17(32.1)$ & 0.907 \\
\hline Basis disease & $13(31.0)$ & $5(9.4)$ & 0.690 \\
Hypertension, n (\%) & $3(7.1)$ & $17(32.1)$ & 0.375 \\
Coronary heart disease, $\mathrm{n}(\%)$ & $10(23.8)$ & $6(11.3)$ & 0.452 \\
COPD, n (\%) & $7(16.7)$ & $9(17.0)$ & 0.488 \\
Chronic nephrosis, n (\%) & $5(11.9)$ & $13(24.5)$ & 0.523 \\
Diabetes, $\mathrm{n}(\%)$ & $8(19.0)$ & $11(20.8)$ & 0.722 \\
Immunosuppression, n (\%) & $10(23.8)$ & $17(32.1)$ & 0.123 \\
Tumor, n (\%) & $20(47.6)$ & $5(9.4)$ & 0.988 \\
Smoking, n (\%) & $4(9.5)$ & & \\
Drinking, n (\%) & & & \\
\hline
\end{tabular}

2 Note: The chi-square test was utilized to calculate the difference between the two groups. $P<$

30.05 was considered statistically significant. Abbreviations: COPD: chronic obstructive

4 pulmonary disease. 


\section{Table 2 (on next page)}

Laboratory examination before treatment, Ventilator parameters, APACHE II score and SOFA score before treatment of two groups.

There were no any differences in laboratory examination, ventilator parameters, APACHE II score and SOFA score before treatment between two groups $(P>0.05)$. 
1 Table 2. Laboratory examination before treatment, Ventilator parameters, APACHE II score and

2 SOFA score before treatment of two groups.

\begin{tabular}{|c|c|c|c|}
\hline & NGS $(n=42)$ & no-NGS (n=53) & $P$ value \\
\hline \multicolumn{4}{|l|}{ Laboratory examination before } \\
\hline \multicolumn{4}{|l|}{ treatment } \\
\hline PCT (ug/L) & $1.3(0.5,8.4)$ & $2.5(0.3,10.6)$ & 0.516 \\
\hline WBC $\left(10^{9} / \mathrm{L}\right)$ & $10.5(6.4,15.4)$ & $13.1(7.5,15.5)$ & 0.189 \\
\hline $\mathrm{Hb}(\mathrm{g} / \mathrm{L})$ & $109(85,130)$ & $105(84,129)$ & 0.932 \\
\hline $\operatorname{PLT}\left(10^{9} / \mathrm{L}\right)$ & $159(84,205)$ & $154(112,197)$ & 0.780 \\
\hline $\operatorname{Scr}(\mu \mathrm{mol} / \mathrm{L})$ & $78(64,201)$ & $97(64,121)$ & 0.515 \\
\hline T.Bil $(\mathrm{mmol} / \mathrm{L})$ & $11.8(5.2,17.2)$ & $14.4(7.8,21.1)$ & 0.071 \\
\hline $\operatorname{ALT}(\mathrm{IU} / \mathrm{L})$ & $28(20,47)$ & $27(20,45)$ & 0.612 \\
\hline $\mathrm{Alb}(\mathrm{g} / \mathrm{L})$ & $28.0(23.6,31.6)$ & $28.2(24.8,32.6)$ & 0.880 \\
\hline APTT (sec) & $35.6(31.0,44.7)$ & $34.7(26.4,48.1)$ & 0.614 \\
\hline NT-proBNP (pg/ml) & $652(236,2747)$ & $656(311,2066)$ & 0.482 \\
\hline $\mathrm{Lac}(\mathrm{mmol} / \mathrm{L})$ & $1.6(1.4,2.9)$ & $1.7(1.2,2.5)$ & 0.763 \\
\hline \multicolumn{4}{|l|}{ Ventilator parameters } \\
\hline $\mathrm{FiO}_{2}$ & $0.8(0.6,1.0)$ & $0.6(0.5,0.8)$ & 0.992 \\
\hline Peep & $10(8,15)$ & $8(6,12)$ & 0.272 \\
\hline OI & $124(76,177)$ & $156(108,194)$ & 0.996 \\
\hline APACHE II score before treatment & $22(18,26)$ & $21(17,26)$ & 0.500 \\
\hline SOFA score before treatment & $7(5,8)$ & $7(4,8)$ & 0.875 \\
\hline
\end{tabular}

3 Note: the measured data of patients' physiological indicators in the above table were shown by

4 median (interquartile range). $P<0.05$ was considered statistically significant. Abbreviations:

5 PCT: Procalcitonin; WBC: White blood cell; Hb: Hemoglobin; PLT: Platelet count; Scr: Serum

6 creatinine; T.Bil: Total bilirubin; ALT: Alanine aminotransferase; Alb: Albumin; APTT:

7 Activated partial thromboplastin time; NT-proBNP: N-terminal Pro-Brain Natriuretic Peptide; 
8 Lac: Lactate; FiO2: Fraction of inspiration O2; Peep: positive end-expiratory pressure; OI:

9 Oxygenation Index; APACHE-II: Acute physiology and chronic health evaluation-II; SOFA:

10 Sequential Organ Failure Assessment. 


\section{Table 3(on next page)}

ICU special treatment of two groups.

There were no any differences in ICU special treatment between two groups $(P>0.05)$. 
1 Table 3. ICU special treatment of two groups.

\begin{tabular}{lccc}
\hline & NGS (n=42) & no-NGS (n=53) & $P$ value \\
\hline Use of vasoactive agent, n (\%) & $24(57.1)$ & $30(56.6)$ & 0.958 \\
CRRT, n (\%) & $9(21.4)$ & $7(13.2)$ & 0.288 \\
ECMO, n (\%) & $6(14.3)$ & $3(5.7)$ & 0.177 \\
Prone positioning, n (\%) & $10(23.8)$ & $11(20.8)$ & 0.722 \\
\hline
\end{tabular}

2 Note: The chi-square test was utilized to calculate the difference between the two groups. $P<$

30.05 was considered statistically significant. Abbreviations: ICU: Intensive care unit; CRRT:

4 continuous renal replacement therapy; ECMO: Extracorporeal membrane oxygenation.

5 


\section{Table 4 (on next page)}

Comparison of outcomes between NGS and no-NGS groups.

The primary outcome: There was a significant difference in 28-day all-cause mortality between the two groups $(P=0.006)$. The secondary outcome: There was no significant difference in the length of stay in the ICU, the duration of mechanical ventilation, ECMO, prone position ventilation, or the cost of the ICU stay between the two groups $(P>0.05)$. 
1 Table 4. Comparison of outcomes between NGS and no-NGS groups.

\begin{tabular}{lccc}
\hline & NGS $(\mathrm{n}=42)$ & no-NGS (n=53) & $P$ value \\
\hline The primary outcome & & & \\
28-day all-cause mortality & $9(21.4 \%)$ & $26(49.1 \%)$ & $\mathbf{0 . 0 0 6 *}$ \\
\hline The secondary outcomes & $12(7,20)$ & $11(8,15)$ & 0.719 \\
Length of stay in ICU (d) & $240(144,353)$ & $216(134,311)$ & 0.810 \\
Duration of mechanical ventilation (h) & $15(11,18)$ & $10(10,23)$ & 0.500 \\
Duration of ECMO (d) & $89(63,117)$ & $96(71,121)$ & 0.345 \\
Duration of prone position ventilation & & & \\
$\quad(h)$ & $82.3(55.1,211.1)$ & $98.9(68.9,141.1)$ & 0.297 \\
\hline
\end{tabular}

2 Note: The chi-square test was utilized to calculate the difference between the two groups in

3 the primary outcome. The t-test was utilized to calculate the difference between the two groups

4 in the secondary outcome. The measured data of patients' outcomes in the above table were

5 shown by median (interquartile range). $P<0.05$ was considered statistically significant. 


\section{Table 5 (on next page)}

Cox multivariate analysis of prognosis of patients with ARDS.

The NGS group had a better prognosis than no-NGS group $(P=0.005)$. Those with a shorter stay in the ICU $(P=0.037)$, and lower APACHE II before treatment $(P=0.016)$ and SOFA scores before treatment $(P=0.003)$ had a better prognosis. 
1 Table 5. Cox multivariate analysis of prognosis of patients with ARDS.

\begin{tabular}{ccccc}
\hline & HR & Lower .95 & Upper .95 & $P$-value \\
\hline mNGS (yes/no) & 0.263 & 0.105 & 0.663 & $\mathbf{0 . 0 0 5 *}$ \\
Age (yr) & 1.013 & 0.988 & 1.038 & 0.322 \\
Length of stay in ICU (d) & 0.888 & 0.794 & 0.993 & $\mathbf{0 . 0 3 7 ^ { * }}$ \\
APACHE II score before treatment & 1.112 & 1.020 & 1.212 & $\mathbf{0 . 0 1 6 *}$ \\
SOFA score before treatment & 1.339 & 1.105 & 1.622 & $\mathbf{0 . 0 0 3 *}$ \\
Coronary heart disease (yes/no) & 1.660 & 0.556 & 4.958 & 0.364 \\
Bronchiectasis (yes/no) & 1.128 & 0.331 & 3.843 & 0.848 \\
Diabetes (yes/no) & 0.324 & 0.088 & 1.195 & 0.091 \\
Hb (g/L) & 0.993 & 0.980 & 1.006 & 0.284 \\
T.Bil (mmol/L) & 0.999 & 0.987 & 1.012 & 0.882 \\
Be & 1.063 & 0.996 & 1.133 & 0.064 \\
Use of vasoactive agent (yes/no) & 1.443 & 0.587 & 3.548 & 0.424 \\
ECMO (yes/no) & 1.212 & 0.067 & 21.764 & 0.896 \\
Cost in ICU (CNY) & 1.000 & 1.000 & 1.000 & 0.477 \\
\hline
\end{tabular}




\section{Table 6(on next page)}

Comparison of metagenomic NGS results and conventional microbiological tests.

The positive rate of $\mathrm{mNGS}$ virus detection was lower than that of serum antibody detection plus PCR (6.7\% vs. $26.7 \%, P=0.021)$. mNGS was significantly better at detecting bacteria than serological antibody testing plus PCR $(24.4 \%$ vs. $0 \%, P=0.001)$. Further, mNGS was able to detect specific pathogens better than the culture method $(22.2 \%$ vs. $0 \%, P=0.001)$ and serological antibody testing plus PCR ( $22.2 \%$ vs. $2.2 \%, P=0.007)$. Additionally, mNGS was significantly better at the identification of co-infections than serological antibody tests plus PCR $(26.7 \%$ vs. $0 \%, P<0.001)$. Finally, mNGS proved to be significantly better at identifying pathogens than the culture method $(91.1 \%$ VS $62.2 \%, P=0.001)$ and serological antibody testing plus PCR (91.1\% vs. $28.9 \%, P<0.001)$. 
1 Table 6. Comparison of metagenomic NGS results and conventional microbiological tests.

\begin{tabular}{cccccc}
\hline & $\begin{array}{c}\text { Method A } \\
(\mathrm{n}=45)\end{array}$ & $\begin{array}{c}\text { Method B } \\
(\mathrm{n}=45)\end{array}$ & $\begin{array}{c}\text { Method C. } \\
(\mathrm{n}=45)\end{array}$ & $\begin{array}{c}P \text {-value, } \\
\text { A vs. B }\end{array}$ & $\begin{array}{c}P \text {-value, } \\
\text { A vs. C }\end{array}$ \\
\hline Only virus, n (\%) & $3(6.7)$ & $0(0.0)$ & $12(26.7)$ & 0.24 & $\mathbf{0 . 0 2 1 *}$ \\
Only bacterial, n (\%) & $11(24.4)$ & $15(33.3)$ & $0(0.0)$ & 0.486 & $\mathbf{0 . 0 0 1 *}$ \\
Only fungus, n (\%) & $5(11.1)$ & $5(50.0)$ & $0(0.0)$ & 1 & 0.056 \\
Special pathogen, n & $10(22.2)$ & $0(0.0)$ & $1(2.2)$ & $\mathbf{0 . 0 0 1 *}$ & $\mathbf{0 . 0 0 7 *}$ \\
$(\%)$ & $12(26.7)$ & $8(17.8)$ & $0(0.0)$ & 0.311 & $<\mathbf{0 . 0 0 1 *}$ \\
Co-infection, n (\%) & & & & \\
Overall Positive, n (\%) & $41(91.1)$ & $28(62.2)$ & $13(28.9)$ & $\mathbf{0 . 0 0 1 *}$ & $<\mathbf{0 . 0 0 1 *}$ \\
\hline
\end{tabular}

2 Note: Method A: mNGS; Method B: Culture; Method C: Serological antibody test plus PCR.

3 The Chi-square test was utilized to calculate the difference between the two groups. $P<0.05$ was 4 considered statistically significant. 
Figure 1

Analysis of 28-day survival curves of patients in the NGS group and no-NGS group.

The 28-day survival was significantly higher in the NGS group than in the no-NGS group $(\mathrm{HR}=2.41,95 \% \mathrm{Cl}: 1.21-4.17, P=0.01)$

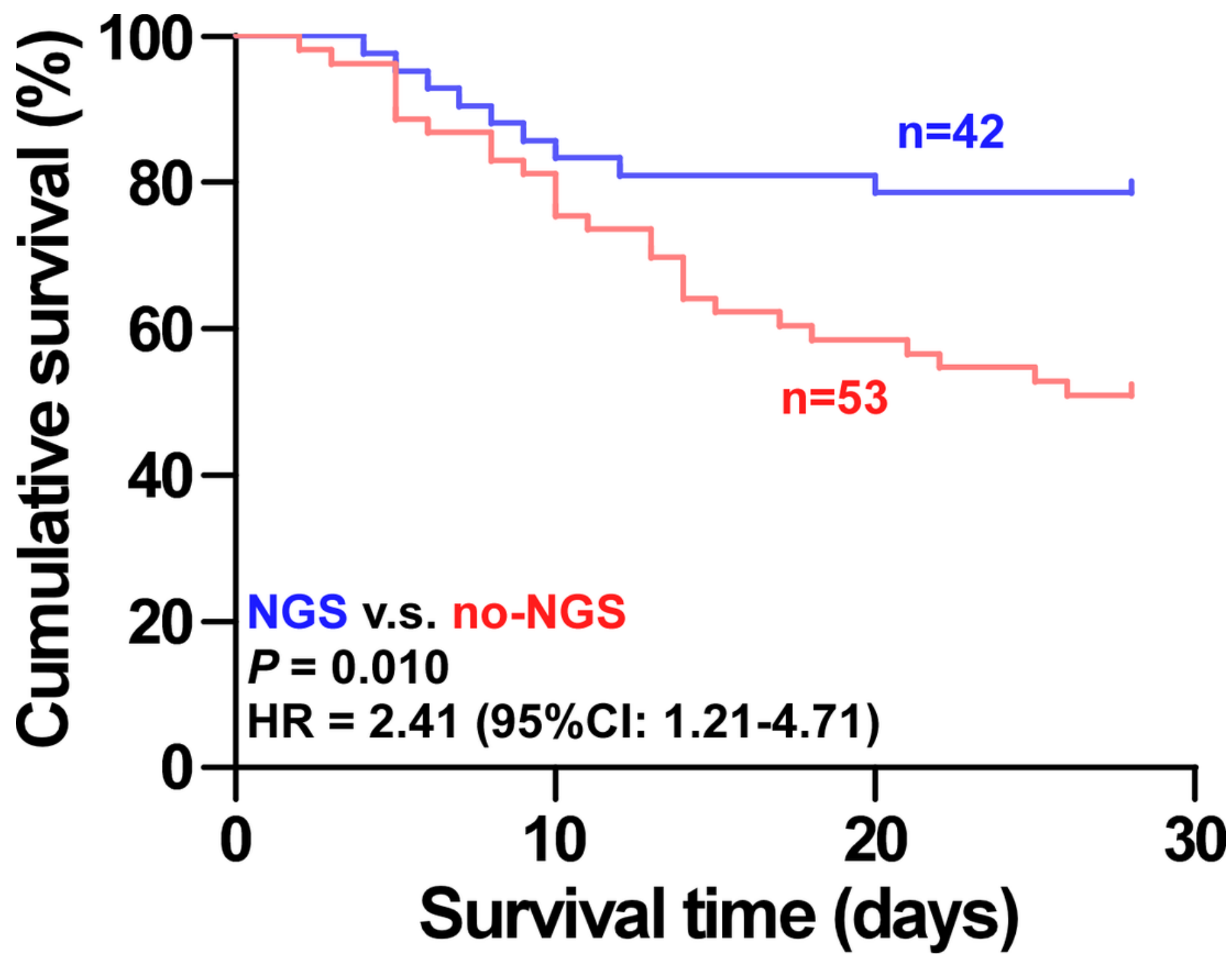




\section{Figure 2}

The consistent analysis comparing culture and mNGS pathogen detection in the NGS group.

Identified pathogens (31.1\%) in the NGS group were consistent, $15.6 \%$ were partially consistent, and $53.3 \%$ were completely inconsistent. In the inconsistent ones, $62.5 \%$ were negative for the culture method, while $8.3 \%$ were negative for mNGS, and $29.2 \%$ were mismatched.

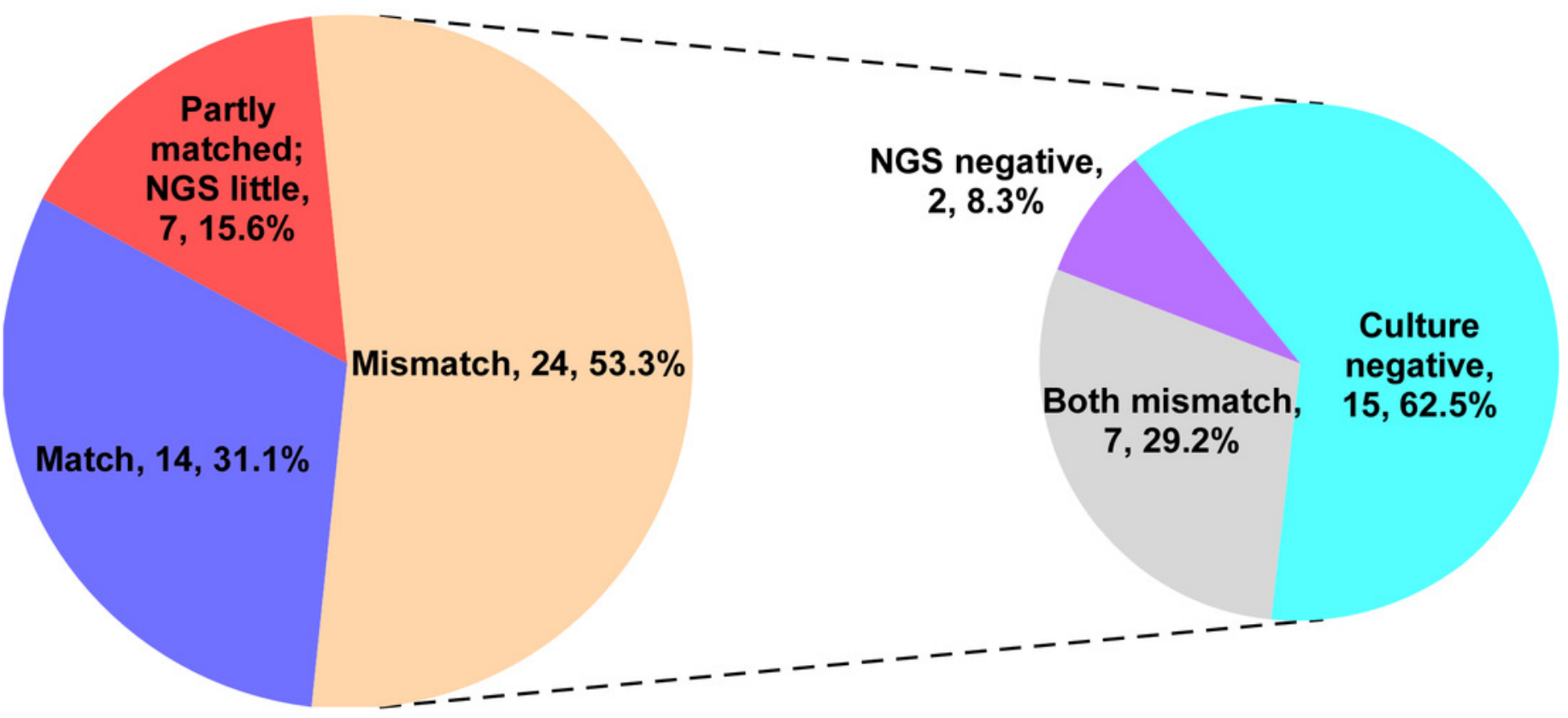




\section{Figure 3}

Coverage spectrum of empirical antimicrobial therapy for pathogen detection results in two groups.

A: In the NGS group, 30 patients (71.4\%) did not cover all the microbial detected by mNGS in the initial empirical antimicrobial treatment. Thus, antimicrobial regimen needs to be modified accordingly based on the mNGS results. B: In the no-NGS group, empirical antimicrobial treatment that could not cover the detected microbials was found in 23 patients (43.4\%), according to the results of traditional microbiological testing, and they were necessary to adjust the anti-infection program.

A

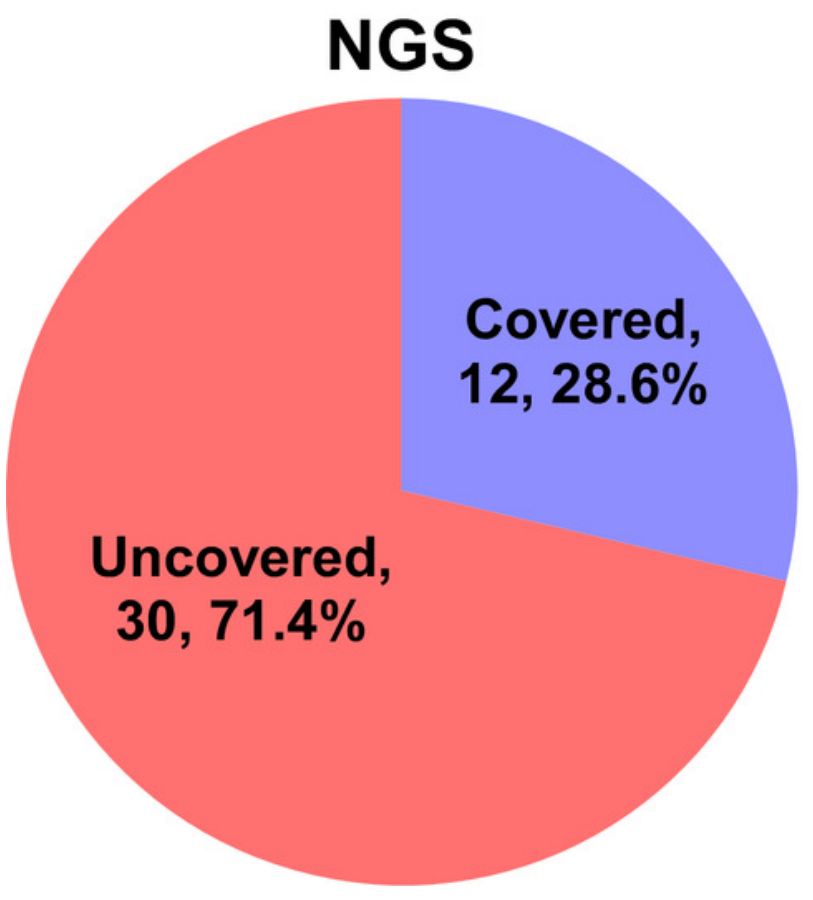

B

\section{no-NGS}

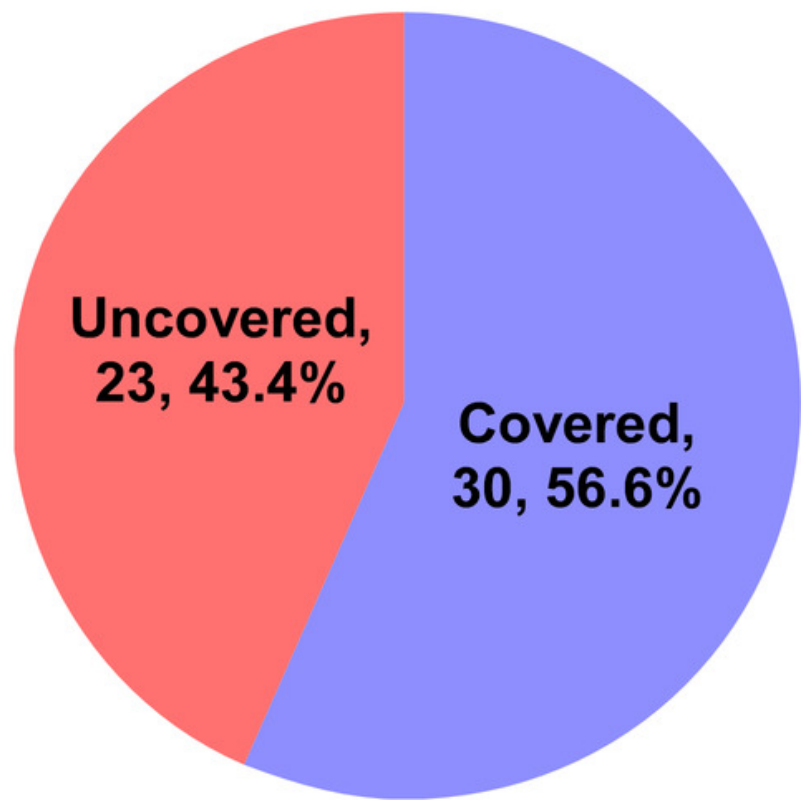


Figure 4

APACHE II and SOFA scores of the two groups.

A: The NGS group had a lower APACHE II score than that in the no-NGS group after 7 days of treatment $(P=0.041)$. B: There was no significant difference in SOFA score during 7 days between two groups $(P \square 0.05)$.

A

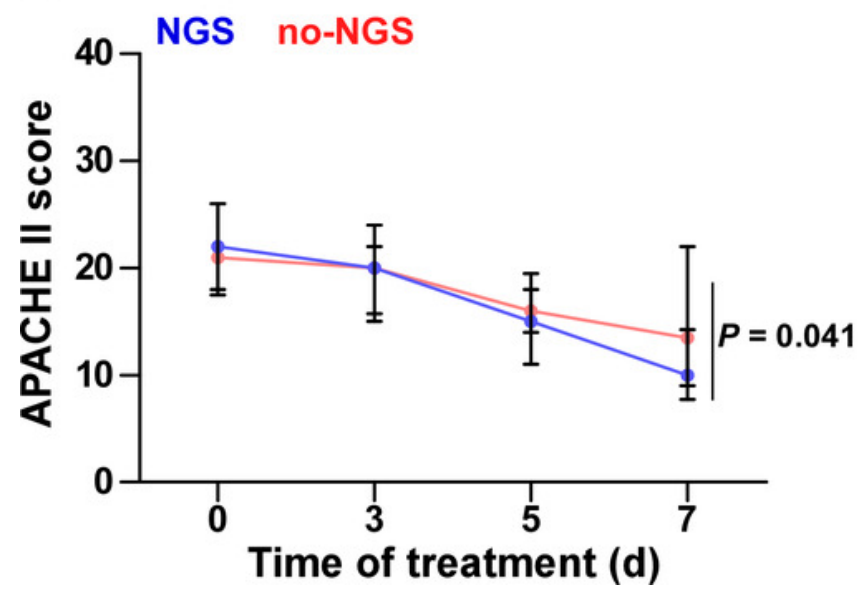

B

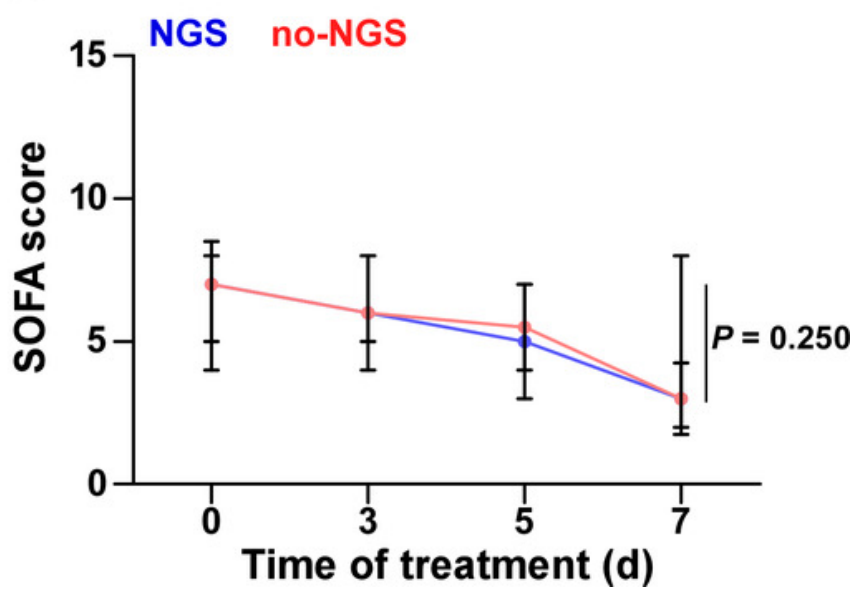


Figure 5

Clinical data of 21 immunosuppressed patients with NGS and no-NGS were compared.

The NGS group had shorter length of stay in the ICU (A) $(P=0.023)$, shorter ventilation time (B) $(P=0.030)$, and less cost in ICU (C) $(P=0.004)$ than those in the no-NGS group of immunosuppressed patients.
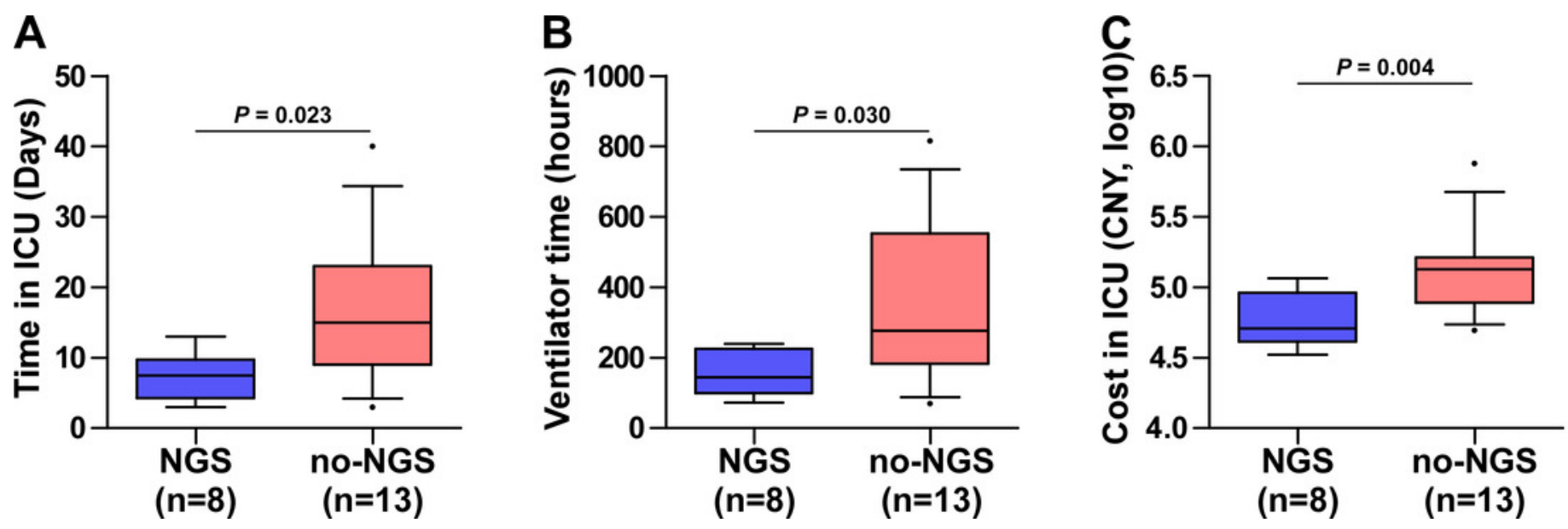